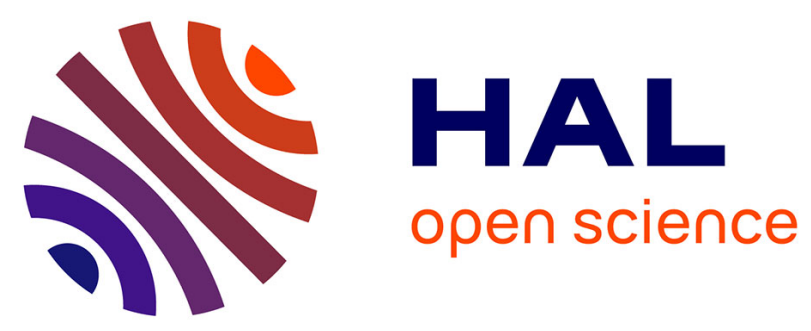

\title{
Never Trust a Cable Bearing Echoes: Understanding Ambiguities in Time-Domain Reflectometry Applied to Soft Faults in Cables
}

Andréa Cozza

\section{- To cite this version:}

Andréa Cozza. Never Trust a Cable Bearing Echoes: Understanding Ambiguities in Time-Domain Reflectometry Applied to Soft Faults in Cables. IEEE Transactions on Electromagnetic Compatibility, 2018, 10.1109/TEMC.2018.2830404 . hal-01774980

\section{HAL Id: hal-01774980}

https://hal-centralesupelec.archives-ouvertes.fr/hal-01774980

Submitted on 24 Apr 2018

HAL is a multi-disciplinary open access archive for the deposit and dissemination of scientific research documents, whether they are published or not. The documents may come from teaching and research institutions in France or abroad, or from public or private research centers.
L'archive ouverte pluridisciplinaire HAL, est destinée au dépôt et à la diffusion de documents scientifiques de niveau recherche, publiés ou non, émanant des établissements d'enseignement et de recherche français ou étrangers, des laboratoires publics ou privés. 


\title{
Never Trust a Cable Bearing Echoes: Understanding Ambiguities in Time-Domain Reflectometry Applied to Soft Faults in Cables
}

\author{
Andrea Cozza
}

\begin{abstract}
Time-domain reflectometry (TDR), the most widely used testing method for fault-detection in cables, is tested against soft faults of increasing severity. The intensity of TDR echoes is proven to be an unreliable estimator of fault severity, since both the bandwidth of testing signals and the length of the fault have a strong impact on the results. Moreover, it is proven that faults of very different severity may generate virtually identical echoes making it impossible to assess how critical a fault is. Simple frequency-domain estimators are introduced, based on models of a fault reflectivity, leading to minimum requirements for the test bandwidth in order to accurately identify soft faults. As a practical consequence, it is concluded that only faults above a critical length can be accurately identified, implying that no clear decision can be taken about shorter faults.
\end{abstract}

Index Terms-Transmission line and cable testing, fault identification, soft impedance faults.

\section{INTRODUCTION}

$\mathbf{T}$ IME-DOMAIN reflectometry (TDR) has been used for decades as an effective method for detecting the presence of unwanted discontinuities in cables [1], e.g., looking for short- or open-circuits, i.e., hard faults, that could endanger the operability of a cable network. During the last decade, a number of TDR-based methods have been introduced and applied to detect non-critical local faults [2]-[4], often referred to as soft faults, e.g., partial removal of cable conductors/coatings, and any local modification that, while still allowing the use of the cable, could eventually develop into a hard fault. These faults can be ascribed to a local change in the cable characteristic impedance, passing from its nominal value $Z_{o}$ to $Z_{F}$.

At first look, extending the application of TDR from hard to soft faults might appear as warranted and smooth. In fact, it is here shown to neglect physical limitations intrinsic to the way a soft fault responds to testing signals. Previous works in the literature have not taken into account the deep differences between the behavior of hard and soft faults.

This letter formally proves that soft faults tested in their lower-frequency range display two features that make interpreting TDR results an ill-posed problem. The first is the observation that they generate echoes proportional to the bandwidth over which they are tested. The second is the fact that echoes are proportional to both their severity and their

Andrea Cozza is with GeePs, Group of electrical engineering - Paris UMR CNRS 8507, CentraleSupelec, Univ Paris-Sud, Sorbonne Universités, UPMC Univ Paris 06, 11 rue Joliot Curie, Plateau de Moulon 91192 Gif sur Yvette CEDEX.

Contact e-mail: andrea.cozza@ieee.org length. These two properties put in jeopardy any attempt at using the intensity of echoes from soft fault as a measure of their severity.

The letter presents experimental evidence that echoes from soft faults, while yielding precious information about their presence and location, should not be taken as a proxy of their severity. In particular, four different faults are described that have the special property of presenting very similar TDR echoes, even though the are affected by local mismatches in a $1 \div 5$ range.

These results have practical importance as any earlywarning system requires the availability of criteria for deciding whether a soft fault has reached a critical level calling for intervention. It is then argued that fault severity can be estimated only if the fault is longer than a minimum critical length, related to the frequency range over which a cable is tested.

\section{FAULT MODELS AND IMPLICATIONS}

Consider a testing signal $p(t)$ with a spectral content covering the bandwidth $B_{T}$ in base band. When applied to a cable under test, a fault at a distance $d$ would generate an echo $e(t)$, whose Fourier spectrum is given by

$$
E(\nu)=\Gamma_{F}(\nu) P(\nu) \exp (-\mathrm{j} 4 \pi \nu \tau),
$$

with $\nu$ the frequency, $\tau=d / v$ the time-of-flight or propagation delay between the testing port and the fault and $v$ the propagation speed in the cable, assumed to be weakly dispersive over $B_{T}$. Capital quantities stand for Fourier spectra.

The reflectivity $\Gamma_{F}(\nu)$ of a fault of length $w$ was shown to be [5]

$$
\Gamma_{F}(\nu)=\frac{2 \mathrm{j} \Gamma_{o} \mathrm{e}^{-\mathrm{j} k w} \sin (k w)}{1-\Gamma_{o}^{2} \mathrm{e}^{-\mathrm{j} 2 k w}}
$$

with $k=2 \pi \nu / v$ the propagation constant of the cable and $\Gamma_{o}=\left(Z_{F}-Z o\right) /\left(Z_{F}+Z_{o}\right)$ the surge impedance mismatch associated to the fault, which will be used throughout this letter as a measure of the fault severity. Most faults have a limited extension, with $w$ shorter than a few centimeters, while testing signals have spectra usually below the $\mathrm{GHz}$ range, because of typical higher losses in the microwave region in commonly used cables and increased costs in testing instrumentation. Eq. (2) can be approximated, for $k w \ll 1$, as

$$
\Gamma_{F}(\nu) \simeq \mathrm{j} 4 \pi \nu(h / v) \mathrm{e}^{-\mathrm{j} k w},
$$


with

$$
h=\Gamma_{o} w /\left(1-\Gamma_{o}^{2}\right) .
$$

As a result, the fault echo reads

$$
e(t)=(2 h / v) \dot{p}(t-2 \tau-T),
$$

with $T=w / v$ and $\dot{p}(t)$ the first time derivative of $p(t)$. This derivative approximation is expected to hold for $\nu \lesssim f_{o}$, where

$$
f_{o}=\frac{1}{2 \pi T} \frac{1-\Gamma_{o}^{2}}{1+\Gamma_{o}^{2}}
$$

is the critical frequency of the fault [5].

Eq. (5) has direct practical implications for TDR tests of soft faults. First, assume that two test signals were used, $p(t)$ and $p^{\prime}(t)$, with bandwidths $B_{T}$ and $B_{T}^{\prime}=\alpha B_{T}$, respectively, such that $p^{\prime}(t)=p(\alpha t)$. The linear approximation (5) has

$$
e^{\prime}(t)=\alpha(2 h / v) \dot{p}(\alpha t-2 \tau-T),
$$

implying that the two TDR echoes $e(t)$ and $e^{\prime}(t)$ are related by a scale-invariance property

$$
e^{\prime}(t)=\alpha e(\alpha t) .
$$

The amplitude of fault echoes is therefore bandwidth dependent, with intensity proportional to the test bandwidth

$$
\max _{t}\left|e^{\prime}(t)\right| / B_{T}^{\prime}=\max _{t}|e(t)| / B_{T} .
$$

Hence, the intensity of TDR echoes should not be confused as a direct indication of fault severity, as even severe faults can result into weak echoes as soon as they are tested at sufficiently low frequencies. Note that the bandwidth dependence is not an artefact due to a changing energy of $p(t)$ as $B_{T}$ increases, but is rather explained by the fact that cable faults respond more strongly at higher frequencies, as clear from (3).

The second implication of (5) is that $h \simeq \Gamma_{o} w$ controls the intensity of a fault response, rather than $\Gamma_{o}$ alone. Therefore, identical responses may be witnessed for faults of different severity, as long as they share the same value of $h$. Since the length of a fault is unknown, it is not possible to estimate $\Gamma_{o}$ once $h$ is accessible. This observation calls into question any attempt at estimating a fault severity $\Gamma_{o}$ from the echoes it produces.

In order to estimate fault parameters $\Gamma_{o}$ and $w,(2)$ requires that testing signals probe the frequency region where $\Gamma_{F}(\nu)$ no longer scales with $\nu$. Eq. (2) predicts that $\Gamma_{F}(\nu)$ must be periodic, with a period inversely proportional to $w$. Therefore $w$ can be retrieved by accessing to the frequency $\nu^{\star}$ for which $\left|\Gamma_{F}(\nu)\right|$ reaches its maximum value for the first time, expected from (2) to occur at

$$
\nu^{\star}=v / 4 w,
$$

for which

$$
\left|\Gamma_{F}\left(\nu^{\star}\right)\right|=\frac{2\left|\Gamma_{o}\right|}{\left(1+\Gamma_{o}^{2}\right)} \simeq 2\left|\Gamma_{o}\right|,
$$

straightforwardly leading to estimators

$$
\begin{aligned}
\left|\hat{\Gamma}_{o}\right| & \simeq\left|\Gamma_{F}\left(\nu^{\star}\right)\right| / 2 \\
\hat{w} & =v / 4 \nu^{\star} .
\end{aligned}
$$

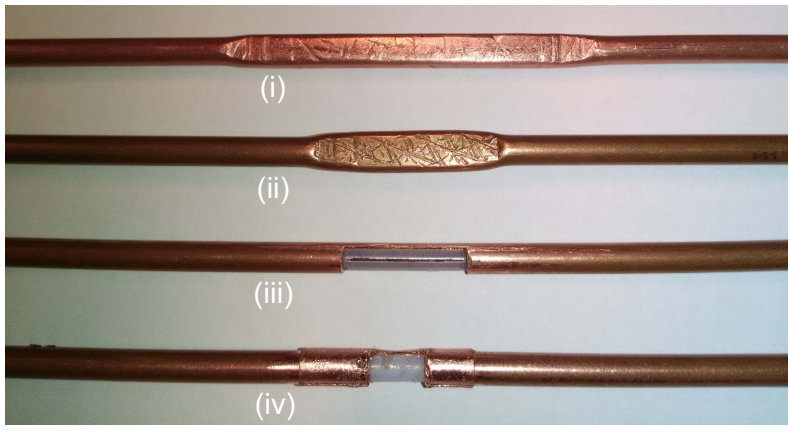

Figure 1. The four cable faults tested, sorted from the least (i) to the most severe (iv).

By the same token, if a cable is tested over a bandwidth $B_{T}$, then only those faults whose length roughly exceeds

$$
w \gtrsim v / 4 B_{T}
$$

can have their severity estimated, as the region around the peak of $\Gamma_{F}(\nu)$ would otherwise not be accessible.

Estimators (12) provide the simplest way of assessing the severity of an impedance fault, and leave no room for ambiguity as they are based on data collected over the maximum of $\left|\Gamma_{F}(\nu)\right|$. At the same time, the two fault parameters $\left|\Gamma_{o}\right|$ and $w$ could be estimated by fitting (2) to the data at frequencies below $\nu^{\star}$. The main issue with such an approach is that a nonlinear regression would be involved, which is known to require a close starting guess in order to be successful. As a matter of fact, as $B_{T} / \nu^{\star}$ decreases, (2) will converge to (3), and make fault identification an ill-posed problem. Therefore, the need to access $\nu^{\star}$ should not be regarded as a firm requirement, but rather as a broad criterion that enables fault identification.

\section{EXPERIMENTAL VALIDATION}

Experimental tests were carried out in order to validate the previous predictions of ambiguity in TDR echoes, as well as for testing the accuracy of estimators (12).

Four different faults were created on identical cables made of semi-rigid coaxial lines of $50 \Omega$ nominal impedance, each $30 \mathrm{~cm}$ long. The nominal propagation speed was estimated from measurements to be $v=2.179 \times 10^{8} \mathrm{~m} / \mathrm{s}$. The four faults, shown in Fig. 1, present an increasing severity: (i) is a crushed portion of cable, (ii) is similar but with a higher pressure applied, (iii) has half of its transversal section cut out, exposing its inner conductor, while (iv) has its outer conductor nearly completely removed, but for a $2 \mathrm{~mm}$ wide strip.

The length $w$ of each fault was optimized, through successive trials, in order to obtain similar low-frequency responses, with the aim of verifying that similar TDR echoes would be observed. Each cable was tested over a $6 \mathrm{GHz}$ frequency range, in order to estimate the fault parameters $\Gamma_{o}$ and $w$ by fitting (2) to experimental results over a wide frequency span. These results served as references for the validation of the estimators proposed in (12).

Measurements were carried out by means of a vector network analyzer (VNA), model Rohde \& Schwarz ZVB8, connecting each cable to the VNA and measuring the $S_{11}$ 


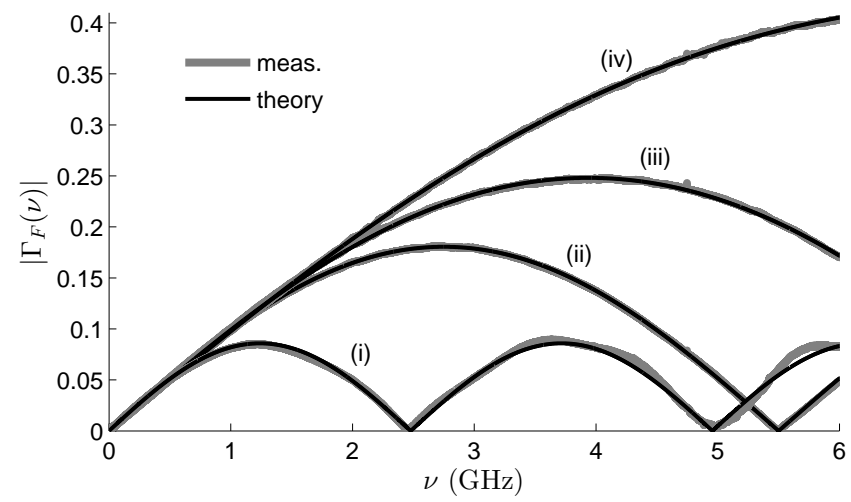

Figure 2. Frequency-domain responses of the four faults in Fig. 1.

parameter while closing the far-end of the cable with a matched load, in order to reduce reflections from the cable end. The rationale behind this choice was to allow a very compact setup with faults on short cables of just $30 \mathrm{~cm}$, without requiring longer cables to be tested, where echoes from faults and terminations would unlikely overlap. Fig. 2 shows the fault responses in the frequency domain, together with those obtained by fitting (2) to the data. The fault parameters associated to these optimal fits are given in Table I and confirm that the four cables are affected by faults of increasing severity, while sharing close values of $|h|$.

All TDR signals shown in the rest of this paper were computed by post-processing frequency-domain data, since such approach let all possible parameter combination to be explored from a single set of results thanks to (1). The spectrum $P(\nu)$ of the test pulse was chosen to be a Kaiser window, with a parameter $\beta=10$. This choice provides a nearly optimal compromise between time resolution and bandwidth, while ensuring a side-lobe level below $-60 \mathrm{~dB}$, limiting the risk of interpreting ripples in the signals as partial reflections from discontinuities. The effective quadratic bandwidth for this pulse is $B_{\text {eq }} \simeq 0.4 B_{T}$.

Table I

FAULT PARAMETERS ESTIMATED FROM MEASUREMENT RESULTS: $w$ STANDS FOR THE GEOMETRICAL LENGTH OF THE FAULT; THE REST OF THE RESULTS WERE OBTAINED BY FITTING (2) OVER A $6 \mathrm{GHZ}$ BANDWIDTH AND WITH THE ESTIMATORS (12).

\begin{tabular}{|c|c|c|c|c|c|c|c|}
\cline { 3 - 8 } \multicolumn{2}{c|}{} & \multicolumn{3}{c|}{ Wide-band fit of (2) } & \multicolumn{3}{c|}{ Estimators (12) } \\
\hline \multirow{2}{*}{ Fault } & $w$ & $\left|\Gamma_{o}\right|$ & $w$ & $|h|$ & $\nu^{\star}$ & $\left|\hat{\Gamma}_{o}\right|$ & $\hat{w}$ \\
\cline { 2 - 8 } & $(\mathrm{mm})$ & & $(\mathrm{mm})$ & $(\mathrm{mm})$ & $(\mathrm{GHz})$ & & $(\mathrm{mm})$ \\
\hline \hline (i) & 44.1 & 0.042 & 43.9 & 1.85 & 1.24 & 0.043 & 44.9 \\
(ii) & 21.2 & 0.091 & 19.8 & 1.82 & 2.73 & 0.090 & 19.8 \\
(iii) & 14.3 & 0.13 & 13.8 & 1.82 & 3.96 & 0.12 & 13.5 \\
(iv) & 6.5 & 0.22 & 7.2 & 1.67 & $>6$ & $>0.21$ & $<9.1$ \\
\hline
\end{tabular}

\section{REsults}

Fig. 2 shows that the four faults have close frequency responses for $\nu<1 \mathrm{GHz}$, a frequency of interest since typical TDR signals seldom exceed it. At much higher frequencies the four $\left|\Gamma_{F}(\nu)\right|$ become very different and could be identified with no difficulty: their periodicity is apparent together with
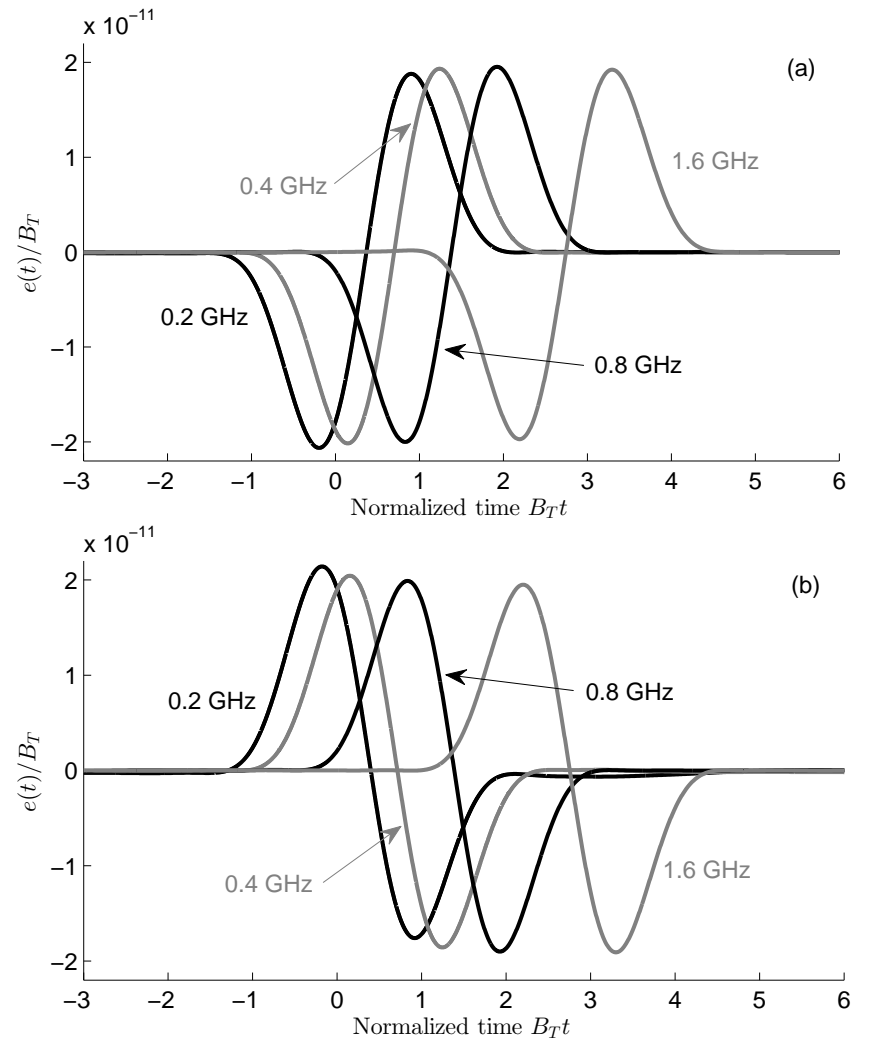

Figure 3. Validation of the scaling rule (8) for the TDR responses of: (a) fault (ii) and (b) fault (iii). Axis are normalized in order to highlight scale invariance with respect to $B_{T}$.

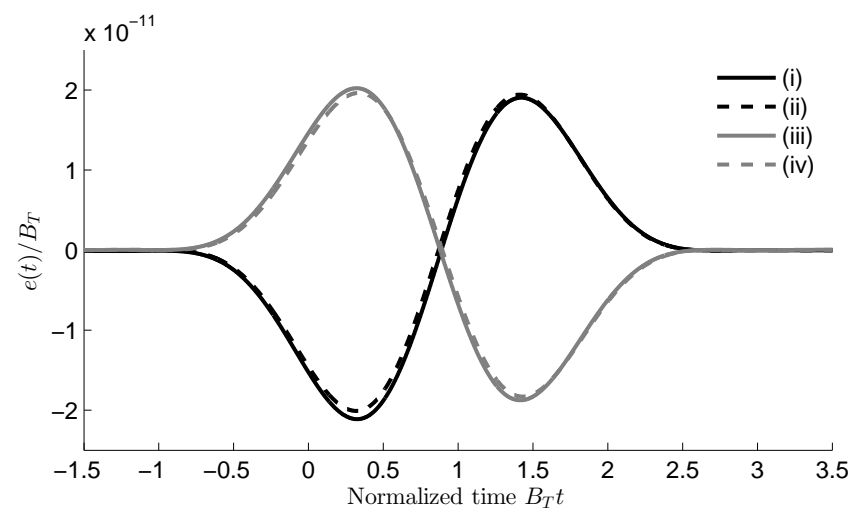

Figure 4. TDR echoes for $B_{T}=500 \mathrm{MHz}\left(B_{e q}=195 \mathrm{MHz}\right.$ ), for the four faults tested.

their peak reflectivity, of which the first occurrence is found at $\nu^{\star}$.

Scale invariance in fault responses, as described in (8) and (9), was first tested by computing the TDR responses for four different choices of the test bandwidth $B_{T}$, going from 0.2 up to $1.6 \mathrm{GHz}$. The results for faults (ii) and (iii), shown in Fig. 3 , were normalized to the bandwidth of each test signal and confirm the validity of (8) and (9), i.e., the amplitude of TDR echoes are linearly proportional to the bandwidth of the test signal. Faults tested for an arbitrary chosen $B_{T}$ can therefore easily mislead the interpretation of TDR results and lead to 
the risk of underestimating the severity of an impedance fault.

Setting now $B_{T}=500 \mathrm{MHz}$, the TDR response of each fault was tested and compared in Fig. 4. As expected from (3) and (5), faults with the same $|h|$ generate echoes of very similar amplitude, despite the fact that a more than five-fold increase in $\Gamma_{o}$ was found in Table I, between faults (i) and (iv). The change in sign between faults (i)-(ii) and (iii)-(iv) is due to the fact that the latter two involve a reduction of the per-unit-length capacitance of the faulty portion, thus resulting in an increase of the line impedance, as opposed to crushed cables.

The practical consequences of these results are clear: when testing faults in their lower frequency range, TDR echoes cannot be regarded as a reliable estimator of fault severity, and it is therefore not possible to take any decision on how critical a fault is on the sole basis of the amplitude of its echoes. The two causes for this ambiguity are the scale invariance in $B_{T}$ and the complementary effect of the impedance mismatch $\Gamma_{o}$ and its length $w$.

The severity of each fault can be estimated by extending the frequency range over which tests are carried out, reaching the first peak at $\nu^{\star}$, reported in Table 1. Estimates of the fault parameters $\left|\hat{\Gamma}_{o}\right|$ and $\hat{w}$ were computed applying (12), yielding the results on the right of Table I. The agreement with the parameters found by fitting (2) to the data, as well as the geometrical length of the faults, confirms the feasibility of unambiguous fault identification from frequency-domain data measured at $\nu^{\star}$. For case (iv) only bounds are given, based on data at $6 \mathrm{GHz}$, since $\Gamma_{F}(\nu)$ does not reach any local maximum, but still provides useful information about the fault.

Covering the frequency $\nu^{\star}$ also appears to ensure accurate estimates of $\left|\Gamma_{o}\right|$ from TDR echoes. Fig. 5 proves that the amplitude of TDR echoes indeed converge to $\left|\Gamma_{o}\right|$ as soon as the equivalent tested bandwidth $B_{\text {eq }} \gtrsim \nu^{\star}$. The convergence is clear in Fig. 5(a) for fault (i), as soon as $B_{T}>3 \mathrm{GHz}$, which corresponds to $B_{\mathrm{eq}}=0.4 B_{T}=\nu^{\star}=1.24 \mathrm{GHz}$. Conversely, Fig. 5(b) considered fault (ii) and does not display a complete convergence, since in this case $\nu^{\star}=2.73 \mathrm{GHz}$, which would require $B_{T}>6.8 \mathrm{GHz}$.

These results point to a more effective procedure if TDR data are analyzed in the frequency domain, where it is simpler to infer how close is the maximum reflectivity $\Gamma_{F}\left(\nu^{\star}\right)$, whereas in time domain, convergence can be assessed only by changing the bandwidth $B_{T}$ over which a cable is tested. In fact, TDR will always need $B_{T}$ well above $\nu^{\star}$, because of the fundamental need for a test signal with a tapered spectrum, in order to control ripples in the time domain that might otherwise be interpreted as potential reflections. Similarly, inverse-scattering approaches seem to require wider bandwidths, as for the case of a $10 \mathrm{~cm}$ fault tested up to 8 $\mathrm{GHz}$ in [6], instead of the $0.5 \mathrm{GHz}$ required by (12).

\section{CONCLUSiOnS}

Severity of faults cannot be inferred from their TDR echoes, as their intensity is not univocally related to fault severity: nearly-severed cables can easily result in very weak echoes, while lighter faults can generate stronger echoes if sufficiently
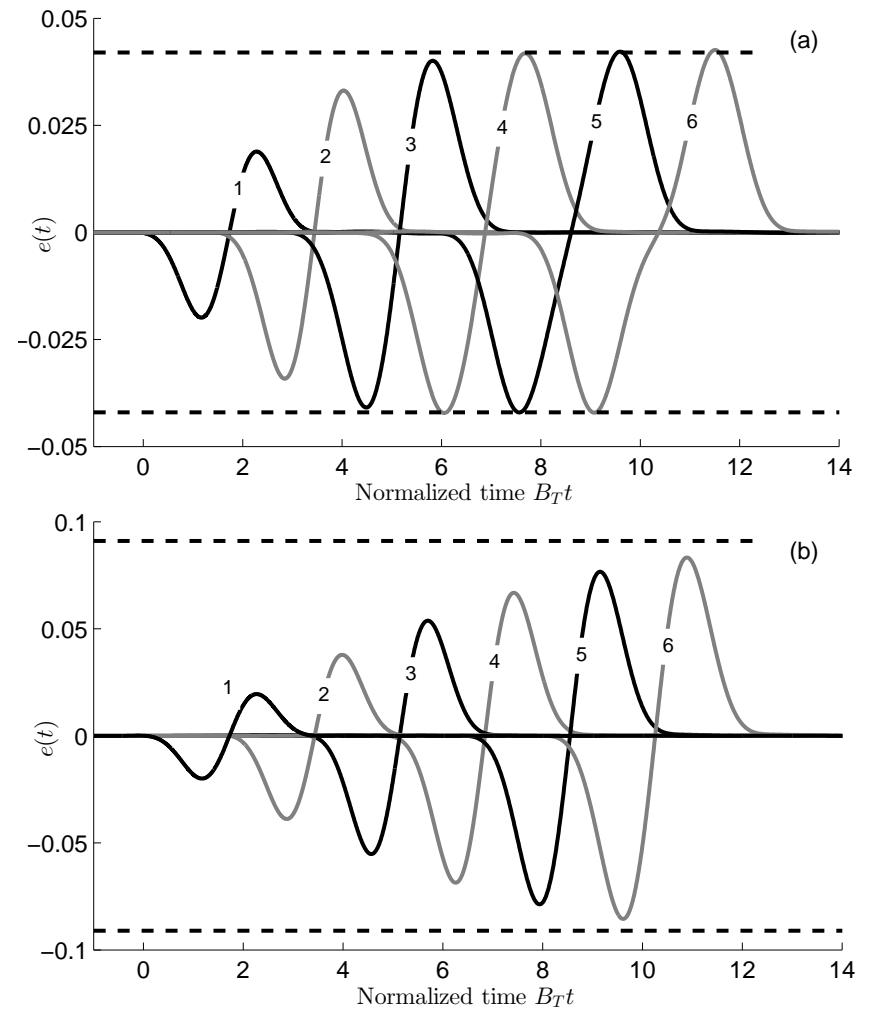

Figure 5. TDR echoes for wide-band test signals, with $B_{T}$ going from 1 up to $6 \mathrm{GHz}$ (see the number on each curve), for : (a) fault (i) and (b) fault (ii). The dashed lines correspond to the fault impedance mismatch $\Gamma_{o}$, towards which converge the peaks of the echoes $e(t)$ as $B_{\text {eq }} \gtrsim \nu^{\star}$.

extended, or if tested at higher frequencies. It is therefore important to start looking at TDR results in a different way and refrain from assuming that weak echoes stand for light faults. More generally, the very idea of associating soft faults to superficial degradations in a cable was shown to be misleading, as a critically damaged cable can still produce very weak echoes. Results confirm that one way of getting around this problem is to carry out tests at sufficiently high frequencies or, as stated by (13), that only sufficiently long faults can be identified.

\section{REFERENCES}

[1] B. M. Oliver, "Time domain reflectometry," Hewlett-Packard Journal, vol. 15, no. 6, pp. 1-7, 1964.

[2] C. Buccella, M. Feliziani, and G. Manzi, "Detection and localization of defects in shielded cables by time-domain measurements with UWB pulse injection and clean algorithm postprocessing," IEEE Trans. Electromagn. Compat., vol. 46, no. 4, pp. 597 - 605, November 2004.

[3] L. Griffiths, R. Parakh, C. Furse, and B. Baker, "The invisible fray: A critical analysis of the use of reflectometry for fray location," IEEE Sensors Journal, vol. 6, no. 3, pp. 697-706, June 2006.

[4] E. Song, Y.-J. Shin, P. Stone, J. Wang, T.-S. Choe, J.-G. Yook, and J. B. Park, "Detection and location of multiple wiring faults via timefrequency-domain reflectometry," IEEE Transactions on Electromagnetic Compatibility, vol. 51, no. 1, pp. 131 -138, feb. 2009.

[5] A. Cozza and L. Pichon, "Echo response of faults in transmission lines: Models and limitations to fault detection," IEEE Trans. Microw. Theory Tech., vol. 64, no. 12, pp. 4155-4164, Dec 2016.

[6] W. Wang, L. Jing, Z. Li, and R. D. Murch, "Utilizing the born and rytov inverse scattering approximations for detecting soft faults in lossless transmission lines," IEEE Trans. Antennas Propag., vol. 65, no. 12, pp. 7233-7243, 2017. 